\title{
An Innovative Concept Gel to Prevent Skin Aging
}

\author{
Adele Sparavigna $^{1}$, Beatrice Tenconi ${ }^{1}$, Ileana Deponti ${ }^{1}$, Francesco Scarci ${ }^{2}$, Maurizio Caserini ${ }^{2}$, \\ Federico Mailland ${ }^{2}$
}

${ }^{1}$ Derming, Clinical Research and Bioengineering Institute, Monza, Italy; ${ }^{2}$ Scientific Department, Polichem S. A., Lugano, Switzerland.

Email: fscarci@polichem.com

Received November $15^{\text {th }}, 2013$; revised December $6^{\text {th }}, 2013$; accepted December $12^{\text {th }}, 2013$

Copyright (C) 2013 Adele Sparavigna et al. This is an open access article distributed under the Creative Commons Attribution License, which permits unrestricted use, distribution, and reproduction in any medium, provided the original work is properly cited.

\begin{abstract}
The study aimed to evaluate the instrumental and clinical properties of an innovative gel formulation for anti-aging treatment. This was an open, non-controlled study, where the eligible subjects, divided into three subgroups according to age, had to perform a single dose application of the gel on the face for a short-term evaluation and a 4-week repeated use, twice a day, for a long-term evaluation. Instrumental and clinical evaluations had to be performed mono-laterally at the level of the face (right or left side according to a predisposed randomization list) in basal conditions $\left(\mathrm{T}_{0}\right), 20$ minutes after the first dose application $\left(\mathrm{T}_{20 \mathrm{~min}}\right)$ and after 2 and 4 weeks of treatment $\left(\mathrm{T}_{2 \mathrm{wks}}-\mathrm{T}_{4 \mathrm{wks}}\right)$. Thirty-three subjects completed the study showing, after only 20 minutes from the $1^{\text {st }}$ product application, a clinically important and statistically significant improvement of crow's feet, skin dullness clinical score and cutaneous microrelief clinical score $(p<0.05)$, after 2 weeks of treatment, an improvement of skin tonicity $(p<0.05)$ and after 4 weeks of treatment having a significant decrease of nasolabial folds clinical score $(\mathrm{p}<0.05)$. The study showed the great efficacy of an innovative antiaging gel in reducing the skin roughness and skin dryness, improving the skin firmness and, in general, providing a global skin rejuvenation.
\end{abstract}

Keywords: Skin; Wrinkles; Anti-Aging

\section{Introduction}

The demand for an anti-aging therapy is increasing day by day and it is not only a prerogative of women as a personal vanity issue; the request is also strong in men, often linked to professional needs. In fact, actors, corporate executives, politicians and showmen (showgirls) need to appear younger and more beautiful. In general, people want to appear younger because a youthful appearance can result in better mental and physical health. The main problem is therefore focused on how to guarantee such expectations as ageing is an unavoidable and irreversible physiological process that leads to some changes in the body [1]. The structure of the skin is made primarily of two layers. The outer layer is the epidermis, made mainly of keratinocytes responsible for the formation of a barrier against environmental damage; the inner one, the dermis constitutes connective tissue and structural components such as collagen (responsible for the skin firmness), elastic fibers (responsible for the skin elasticity) and extracellular matrix (structural component).
The appearance of the skin reflects general health and communicates ethnicity, lifestyle and age. These features are largely determined by skin color, texture, firmness, and smoothness. Aging has a large impact on the quality of all these features [2]. The skin is exposed every day to the environment, oxidative stress and inflammation and for this reason, it undergoes alterations of its structure, leading to the appearance of wrinkles.

Clinically, aged skin may be thin, smooth and with only light wrinkles, but it can also appear thicker with deeply marked wrinkles and with irregular pigmentation $[3,4]$. Skin ageing is characterized by an alteration of structural components of the connective tissue with the consequent formation of skin wrinkles consisting of a disorganization or damage of these skin structures due to a lack of collagen or to its modification, thinning and/or fractioning, caused by the stretching and repeated extension of some areas of the skin, especially the face [5]. The lack of elastin, for example, has an important role in the formation of wrinkles, due to the loss of elasticity [6]. 
A wrinkle is a fold, a ridge or crease in the skin, which appears as a result of the ageing processes such as glycation [7], or promoted by habitual facial expressions, skin type, photo-aging, solar UV irradiation, the effect of gravity, tobacco smoke, hormonal status, pollution, malnutrition, poor hydration and genetic factors $[8,9]$.

There are a lot of treatments boasting the capability to improve wrinkles: pharmaceutical, surgical and cosmetic solutions. These treatments are intended to change the nature of ageing collagen, stretch the skin, fill in the depressions of the skin or paralyse the muscles that cause the wrinkle. Retinoid products, for example, act by inhibiting enzymes from breaking down collagen, but they may produce redness, burning and general discomfort [10]; alpha-hydroxy acids penetrate into the top layer of the skin, producing only subtle improvement, though, and causing a mild and temporary irritation, increasing the skin's sensitivity to the sun and particularly increasing the possibility of sunburn [11]; the anti-aging Serum stimulates the skin to rebuild the collagen and elastin network in the dermis resulting in a renewal of skin structure, improving the skin elasticity and smoothing the wrinkles. The disadvantages, however, are related to the cost and the fact that very often the real composition of these compounds, obtained from an animal source, is not specified. Moreover, reproducibility may not be guaranteed and animal extracts may pose safety issues. In the surgical field, there are several techniques that could result in an excellent improvement but that also produce significant side effects, including scarring and permanent changes in skin color. In other cases, the improvements may last some months then surgery must be repeated. Again, surgical procedures are the opposite to the tissue regeneration process, acting only in an aesthetic way but not in the sense of skin rejuvenation. Cosmetic products include superficial or deeper peels that act in smoothing fine lines and scarring. However, their action is opposite to the tissue regeneration, mostly by trying to improve the skin depressions by lifting the uppermost skin layer. Antioxidants, on the other hand, provide protection from the sun, neutralizing the free radicals responsible for the collagen breakdown: by this mechanism, antioxidants may be very important in the prevention of the further worsening of wrinkle forming. Finally, moisturizers can make wrinkles look temporarily less prominent, keeping the skin hydrated.

As a conclusion, the best product to treat and prevent wrinkle formation should act by regenerating tissues (mostly collagen), by opposing oxidation and by moisturizing skin. In view of all these considerations, a screening was made among different formulations to identify the best possible product for treatment and prevention of wrinkles. That product, P-3086, contains as main ingredient hyaluronic acid, hops and ethanol. P-3086 has been tested in the present study in order to evaluate its instrumental and clinical properties.

\section{Materials and Methods}

The clinical trial was performed in accordance with the GCP-ICH guidelines. The protocol was approved by an independent Ethics Committee. Each subject recruited for the study, signed an informed consent form, after having been fully informed.

\subsection{Subjects and Study Design}

This was an open study aimed to enroll 33 healthy female volunteers, aged 35 - 65 years, stratified in three different groups of 11 women each: 35 - 44 years (Group 1), 45 - 54 years (Group 2) and 55 - 65 years (Group 3). All of them had to present light-moderate facial rhytidosis, without any concomitant disease on the tested area. Each qualifying subject, who met the inclusion/exclusion criteria, performed a single fixed application of the gel on the face (including the submental area) for a short term evaluation and for 4 weeks of repeated use, twice a day (long term evaluation), in the morning and in the evening, with a mild massage.

Instrumental and clinical evaluations were performed, mono-laterally at level of the face (right or left side according to a predisposed randomization list), in basal conditions $\left(\mathrm{T}_{0}\right), 20$ minutes after the first product application $\left(\mathrm{T}_{20 \mathrm{~min}}\right)$ and after 2 and 4 weeks of treatment $\left(\mathrm{T}_{2 \mathrm{wks}}\right.$ $\left.-\mathrm{T}_{4 \mathrm{wks}}\right)$.

\subsection{Instrumental Assessment}

Instrumental measurements were performed in standardized conditions mono-laterally on the face (right or left facial side, randomly) in basal conditions $\left(\mathrm{T}_{0}\right), 20 \mathrm{~min}-$ utes after the first product application $\left(\mathrm{T}_{20 \mathrm{~min}}\right)$ and after 2 and 4 weeks of treatment $\left(\mathrm{T}_{2 \mathrm{wks}}-\mathrm{T}_{4 \mathrm{wks}}\right)$, by means of non-invasive instruments.

The Corneometer CM825 (Courage-Khazaka, Köln, Germany) was used to measure the skin electrical capacitance that is an index of the skin hydration. In order to reduce the variability of the measurement method, for each volunteer, three measures on the same skin area were performed: the adjusted mean was intended as the real measure value.

In order to measure the skin elasticity grade, the Torsiometry was considered as the most effective way, as it is very sensitive to variations of the mechanical properties of the stratum corneum. Skin firmness was therefore measured with the Dermal Torque Meter (Dia-Stron Ltd., UK). This instrument is based on the principle of torsion, given to the skin surface by a probe made of two circles that adhere to the skin through shaped adhesive tapes. While the external circle is still, the internal circle rota- 
tion exerts a constant torsion of skin (torsion time $=1$ second with a torque $=9 \mathrm{mN}^{*} \mathrm{~m}$ ). The instrument measures the torsion angle $(\theta)$ during the mechanical stimulus ("torque on") and after it has ceased ("torque off").

For each of the considered curves ("torque on" and "torque off") the cutaneous rotational ratio relative to define measured times can be measured, obtaining the parameters listed below:

Ue: immediate extensibility ("torque on" at $0.02 \mathrm{sec}$.)

Uf: maximum extensibility ("torque on" at $0.9 \mathrm{sec}$.)

Uv: viscoelasticity

Ur: immediate elastic recovery ("torque off" at 0.02 sec.).

Among methods studied to measure skin elasticity, torsion is demonstrated to be one of the most effective; in fact this method proved to be very sensitive to variation of skin mechanical properties.

The crow's feet area was quantitatively assessed using Primos compact portable device (GFMesstechnik GmbH, Berlin, Germany). The Primos software elaborates 3D representations of skin wrinkles as well as measuring skin principal profilometric parameters in vivo or on skin replicas, according to DIN EN ISO 4228, using a digital stripe projection based on micro mirrors which allow a fast and highly precise measurement data acquisition. Moreover the software compares directly the different images obtained at the different time points as foreseen by the protocol $\left(\mathrm{T}_{0}, \mathrm{~T}_{20 \mathrm{~min}}, \mathrm{~T}_{2 \mathrm{wks}}\right.$ and $\left.\mathrm{T}_{4 \mathrm{wks}}\right)$.

In order to determine the activity of the tested formulation on skin complexion and on skin brightness, the spectrophotometric and colorimetric measurements were performed mono-laterally at the cheekbone level. Skin color was measured by a visible-UV-IR ( $\lambda$ from 300 to $900 \mathrm{~nm}$ ) spectrophotometer (Spectrophotometer DH2000 Top Sensor System) which uses a tungsten halogen lamp and a deuterium lamp, according to CIE (Commission Internationale de l'Eclarage), the main international organization concerned with color and color measurement. Lamps are switched on 30 minutes before instrument use in order to achieve a stable emission. Measurement angle is $90^{\circ}$ (position of the probe on the skin) and measured area is $2 \mathrm{~mm}^{2}$; the used wavelength range of $380-780$ $\mathrm{nm}$ corresponds to the visible light spectrum.

The measurement of the skin brightness was performed by a tri-stimulus colorimeter (Chroma Meter CR$200^{\circledR}$-Minolta), equipped with three special filters to obtain $R, G, B$ values according to CIE. L*a*b* system (CIELAB), the most complete colour-space specified by the CIE (1976), describes all the colours visible to the human eye. The three coordinates of $\mathrm{L}^{*} \mathrm{a} * \mathrm{~b}^{*}$ represent the lightness of the colour $\left(\mathrm{L}^{*}=0\right.$ yields black and $\mathrm{L}^{*}=$ 100 indicates diffuse white), its position between red/ magenta and green ( $a^{*}$, negative values indicate green while positive values indicate magenta) and its position between yellow and blue $\left(b^{*}\right.$, negative values indicate blue and positive values indicate yellow).

Face ptosis determination was carried out by photographic techniques and image analysis; a frontal facial picture of each volunteer was taken at all different time points. In order to ensure comparable images, the pictures were taken using a standardized method, to measure the facial coordinates of each subject in order to identify the relaxed cutaneous area. Image elaboration for face coordinates evaluation was conducted by computer image analysis.

\subsection{Clinical Assessment}

In order to determine the wrinkles grade at level of the nasolabial folds and of the lateral corner of the eye ("crow's feet"), a reference clinical scale was used:

$0=$ No wrinkles, $1=$ Very weak wrinkles, $2=$ Weak wrinkles, 3 = Quite evident wrinkles, $4=$ Evident wrinkles, $5=$ Very evident wrinkles, $6=$ Marked wrinkles, 7 $=$ Very marked wrinkles.

Submental ptosis was scored from 0 (absence of ptosis -very regular oval face) to 5 (very marked ptosis-very irregular oval face) according to a clinical photographic scale.

Based on a photographic scale for the cheek, surface microrelief was evaluated according to following score:

$1=$ very regular: the primary lines present all the same depth. The secondary lines are well demarcated and form star like picture (apexes converge of several triangles).

2 = regular: hiding and loss of secondary lines demarcation. Star-like pictures are still present but with less demarcated secondary lines.

3 = irregular: primary lines irregularity. Strong hiding of lines with low presence of star-like pictures.

4 = very irregular: strong deterioration in the skin. Deep primary lines distortion and loss of secondary lines.

Skin dullness of the overall face was evaluated according to the following score:

1 = luminous skin, 2 = normal skin, 3 = opaque skin, 4 $=$ very opaque skin.

Skin resistance to pinching, resistance to traction and recovery after pinching, were performed at level of cheek (malar region) according to the following score:

$0=$ very important, $1=$ important, $2=$ moderate, $3=$ weak, 4 = very weak.

Skin dryness was evaluated at cheek level (malar region) according to the following score:

$0=$ very hydrated skin, $1=$ hydrated skin, $2=$ normal skin, $3=$ slightly dry skin, $4=$ dry skin, $5=$ very dry skin.

In order to determine the anti-age effect of the study product, the data (mean value) of all considered clinical parameters were combined by means of a particular graph named Spiderming ${ }^{\mathrm{TM}}$, which represents an innova- 
tive and validated method for the description and the visualization of the skin aging grade before and after cosmetic treatments [12]. Spiderming ${ }^{\mathrm{TM}}$ allows visualization of the anti-age effect of a cosmetic product, also in function of the age range and the sex of the included subjects.

\subsection{Statistical Analysis}

The activity of the product at $\mathrm{T}_{20 \mathrm{~min}}, \mathrm{~T}_{2 \mathrm{wks}}$ and $\mathrm{T}_{4 \mathrm{wks}}$ was expressed in absolute values versus baseline $\left(\mathrm{T}_{0}\right)$ for the entire population and for each one of the evaluated groups ( 35 - 44 years, 45 - 54 years, 55 - 65 years).

- Instrumental evaluations were processed by analysis on variance (ANOVA) to test the differences between group means, followed by, in presence of statistically significant results, Dunnett's test used in a multiple comparison procedure aimed at comparing each of a number of treatments with a single control, for clinical evaluations.

- Clinical Evaluations were processed by Friedmann test: a non-parametric statistical test, used to detect differences in treatments across multiple test attempts followed by, in presence of statistically significant results, Dunnett's test.

A comparison between groups was carried out at each considered time as follows:

- ANOVA followed by, in presence of statistically significant results, Tukey test, for instrumental evaluations; it compares the means of every treatment to the means of every other treatment.

- Friedmann test followed by, in presence of statistically significant results, Tukey test, for clinical evaluations.

A difference was considered to be statistically significant at $\mathrm{p}<0.05$.

\section{Results}

All thirty-three subjects enrolled (11 subjects for each study group) completed the study. With regards to the Instrument evaluation and in particular the anti-wrinkle efficacy ("crow's feet" profilometry), the obtained results for the entire population and per separated age groups are summarized in Table 1.

The statistically significant reduction (Dunnett's test $\mathrm{p}$ $<0.05 \mathrm{~T}_{2 \mathrm{wks}} / \mathrm{T}_{4 \mathrm{wks}}$ vs. $\mathrm{T}_{0}$ ) of Rt parameter (total height of the wrinkle) and the clinically important reduction of $\mathrm{Ra}$ (average roughness of the wrinkle) and $\mathrm{Rv}$ (maximum depth of the wrinkle) underlined the important antiwrinkle activity of the study product. Analyzing the three different study groups, it is evident that, particularly on Group 1, but also on Group 2, the anti-wrinkles activity results were very high, significantly superior in Group 1 compared to Group 3 (Ra: Dunnett's test $\mathrm{p}<0.05 \mathrm{~T}_{2 \mathrm{wks}}$
Table 1. Anti-wrinkle efficacy variation vs. baseline on unified data and on separated groups per each parameter.

\begin{tabular}{|c|c|c|c|}
\hline & \multicolumn{3}{|c|}{ Variation vs. Baseline (\%) } \\
\hline & $\mathrm{T} 20 \min$ & $\mathrm{T} 2 \mathrm{wks}$ & T4 wks \\
\hline Ra (average roughness) & $-10 \%$ & $-14.7 \%$ & $-16.5 \%$ \\
\hline Rt (total height) & $-12.5 \%$ & $-21 \%\left(\left(^{*}\right)\right.$ & $-23.8 \%\left(^{*}\right)$ \\
\hline Rv (maximum depth) & $-14.8 \%$ & $-13.8 \%$ & $-20.4 \%$ \\
\hline \multicolumn{4}{|c|}{$\left(^{*}\right)=$ Dunnett test $\mathrm{p}<0.05$} \\
\hline & \multicolumn{3}{|c|}{$\begin{array}{c}\text { Variation vs. Baseline (\%) } \\
\text { Ra (average roughness) }\end{array}$} \\
\hline & $\mathrm{T} 20 \mathrm{~min}$ & T2 wks & T4 wks \\
\hline Group $1(35-44)$ & $-16.5 \%$ & $-23.6 \%\left(^{* \#}\right)$ & $-33 \%\left({ }^{*}\right)$ \\
\hline Group $2(45-54)$ & $-19.2 \%$ & $-21.3 \%\left(^{*}\right)$ & $-26.3 \%\left(^{*}\right)$ \\
\hline Group $3(55-65)$ & $3.6 \%$ & $-1.6 \%$ & $5.1 \%$ \\
\hline \multicolumn{4}{|c|}{$\left(^{*}\right)=$ Dunnett test $\mathrm{p}<0.05\left(^{*}\right)=$ Tukey test $\mathrm{p}<0.05$ vs. Group 3} \\
\hline & \multicolumn{3}{|c|}{$\begin{array}{c}\text { Variation vs. Baseline (\%) } \\
\text { Rt (total height) }\end{array}$} \\
\hline & $\mathrm{T} 20 \min$ & T2 wks & T4 wks \\
\hline Group $1(35-44)$ & $-19.2 \%$ & $-23.7 \%$ & $-34 \%\left(^{*}\right)$ \\
\hline Group $2(45-54)$ & $-20.7 \%$ & $-24.5 \%$ & $-25.2 \%$ \\
\hline Group $3(55-65)$ & $2.2 \%$ & $-14.7 \%$ & $-12.5 \%$ \\
\hline \multicolumn{4}{|c|}{$\left(^{*}\right)=$ Dunnett test $p<0.05$} \\
\hline & \multicolumn{3}{|c|}{$\begin{array}{l}\text { Variation vs. Baseline (\%) } \\
\text { Rv (maximum depth) }\end{array}$} \\
\hline & $\mathrm{T} 20 \mathrm{~min}$ & T2 wks & T4 wks \\
\hline Group $1(35-44)$ & $-21.3 \%$ & $-20.5 \%$ & $-28.8 \%$ \\
\hline Group $2(45-54)$ & $-24.2 \%$ & $-18.9 \%$ & $-28.4 \%$ \\
\hline Group $3(55-65)$ & $-0.4 \%$ & $-2.9 \%$ & $-5.3 \%$ \\
\hline
\end{tabular}

and $\mathrm{T}_{4 \mathrm{wks}}$ vs. $\mathrm{T}_{0}$ for both groups and Rt: Dunnett test $\mathrm{p}<$ $0.05 \mathrm{~T}_{4 \mathrm{wks}}$ vs. $\mathrm{T}_{0}$ for Group 1. Ra: Tukey test $\mathrm{p}<0.05$ $\mathrm{T}_{2 \mathrm{wks}}$ and $\mathrm{T}_{4 \mathrm{wks}}$ Group 1 vs. $\mathrm{T}_{2 \mathrm{wks}}$ and $\mathrm{T}_{4 \mathrm{wks}}$ Group 3).

With regards to the skin electrical capacitance (hydration), a statistically significant increase was achieved already after the first product application (Dunnett's test $\mathrm{p}<0.05 \mathrm{~T}_{20 \mathrm{~min}}$ vs. $\mathrm{T}_{0}$ ), with an improvement of $13.9 \%$ vs. $\mathrm{T}_{0}$; after 2 and 4 weeks of treatment this result remained statistically significant $\left(+11.1 \%\right.$ at $\mathrm{T}_{2 \mathrm{wks}}$ and $+12.9 \%$ at $\mathrm{T}_{4 \mathrm{wks}}$; Dunnett test $\mathrm{p}<0.05$ vs. $\mathrm{T}_{0}$ ), confirming the substantive moisturizing activity of the tested product (Figure 1) mostly in Group 2 (Dunnett's test p $<0.05$ $\mathrm{T}_{20 \mathrm{~min}}, \mathrm{~T}_{2 \mathrm{wks}}$ and $\mathrm{T}_{4 \mathrm{wks}}$ vs. $\mathrm{T}_{0}$ for Group 2) (Table 2).

Data of considered torsiometric parameters of the entire study population are summarized in Table 3: a clinically important and statistically significant reduction (Dunnett's test $\mathrm{p}<0.05$ vs. $\mathrm{T}_{0}$ ) of all torsiometric pa- 
Table 2. Skin electrical capacitance variation for separated groups.

\begin{tabular}{cccc}
\hline & \multicolumn{3}{c}{ Hydration Variation vs. Baseline (\%) } \\
\cline { 2 - 4 } & T20 min & T2 wks & T4 wks \\
\hline Group 1 (35 - 44) & $9.9 \%$ & $2.4 \%$ & $4.2 \%$ \\
Group 2 (45 - 54) & $18.5 \%\left(^{*}\right)$ & $22.6 \%\left(^{*}\right)$ & $16.8 \%\left(^{*}\right)$ \\
Group 3 (55 - 65) & $13.1 \%$ & $8.1 \%$ & $17.9 \%$ \\
\hline
\end{tabular}

$\left({ }^{*}\right)=$ Dunnett test $\mathrm{p}<0.05$.

Table 3. Torsiometric parameters variation on unified data.

\begin{tabular}{cccc}
\hline \multirow{2}{*}{ Parameter } & \multicolumn{3}{c}{ Plastoelasticity Variation vs. Baseline (\%) } \\
\cline { 2 - 4 } & T20 min & T2 wks & T4 wks \\
\hline Ue & $-0.9 \%$ & $-16.6 \%\left(^{*}\right)$ & $-16.8 \%\left(^{*}\right)$ \\
Uf & $-1.5 \%$ & $-19.5 \%\left(^{*}\right)$ & $-17.5 \%\left(^{*}\right)$ \\
Uv & $-2.5 \%$ & $-24.3 \%\left(^{*}\right)$ & $-18.7 \%\left(^{*}\right)$ \\
Ur & $-4.9 \%$ & $-17.1 \%\left(^{*}\right)$ & $-14.6 \%\left(^{*}\right)$
\end{tabular}

( $\left.{ }^{*}\right)$ Dunnett test: $\mathrm{p}<0.05$

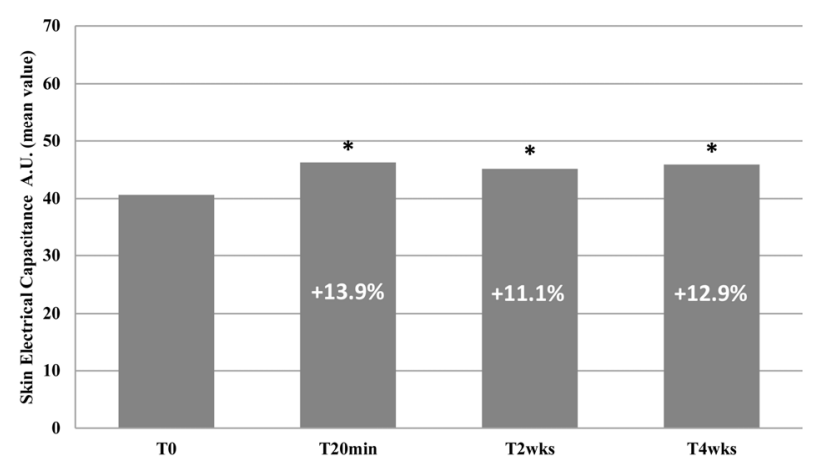

Figure 1. Moisturizing activity on unified data.

rameters starting from $\mathrm{T}_{2 \mathrm{wks}}$, was observed. Even if a trend decrease was present also for Group 1, a more clinically important and statistically redensifying activity was highlighted for Group 2 (Dunnett's test $\mathrm{p}<0.05 \mathrm{Uf}$ $\mathrm{T}_{2 \mathrm{wks}}$ vs. $\mathrm{T}_{0}$ and Ue, Uf, Uv $\mathrm{T}_{4 \mathrm{wks}}$ vs. $\mathrm{T}_{0}$ ) and Group 3 (Dunnett's test $\mathrm{p}<0.05 \mathrm{Ue}, \mathrm{Uf}, \mathrm{Uv}, \mathrm{Ur} \mathrm{T}_{2 \mathrm{wks}} \mathrm{vs}$. $\mathrm{T}_{0}$ and Ue, Uf $\mathrm{T}_{4 \mathrm{wks}}$ vs. $\mathrm{T}_{0}$ ) (Table 4).

The spectrophotometric analysis on pooled data did not reveal any clinically important variation of spectrum total area, but considering the separate groups a trend towards increase of spectrum total area was shown for Group $1\left(+6.2 \% \mathrm{~T}_{20 \mathrm{~min}},+4.3 \% \mathrm{~T}_{2 \mathrm{wks}}\right.$ and $+2 \% \mathrm{~T}_{4 \mathrm{wks}}$ vs. $\mathrm{T}_{0}$ ).

Regarding the optical densitometry, no important variation was shown for skin brightness $\left(\mathrm{L}^{*}\right)$ and redness $\left(a^{*}\right)$, while a statistically significant reduction of skin pigmentation $\left(b^{*}\right)$ was shown starting from $\mathrm{T}_{2 \mathrm{wks}}(-3.3 \%$ at $\mathrm{T}_{2 \mathrm{wks}}$ and $-3.0 \%$ at $\mathrm{T}_{4 \mathrm{wks}}-$ Dunnett's test $\mathrm{p}<0.05$
Table 4. Torsiometric parameters variation for separated groups.

\begin{tabular}{cccc}
\hline \multirow{2}{*}{ GROUP 1 } & \multicolumn{3}{c}{ VARIATION \% vs. T0 } \\
\cline { 2 - 4 } & T20 min & T2 wks & T4 wks \\
\hline Ue & $0.3 \%$ & $-11.1 \%$ & $-15.1 \%$ \\
Uf & $0.6 \%$ & $-11.2 \%$ & $-14.0 \%$ \\
Uv & $1.2 \%$ & $-11.5 \%$ & $-12.1 \%$ \\
Ur & $-10.5 \%$ & $-16.1 \%$ & $-18.5 \%$ \\
GROUP 2 & & & \\
Ue & $2.3 \%$ & $-14.1 \%$ & $-19.9 \%\left(^{*}\right)$ \\
Uf & $1.4 \%$ & $-20.6 \%\left(^{*}\right)$ & $-21.0 \%\left(^{*}\right)$ \\
Uv & $-0.3 \%$ & $-32.2 \%\left(^{*}\right)$ & $-23.1 \%$ \\
Ur & $-1.8 \%$ & $-15.4 \%$ & $-15.6 \%$ \\
GROUP 3 & & & \\
Ue & $-5.0 \%$ & $-23.7 \%\left(^{*}\right)$ & $-15.6 \%\left(^{*}\right)$ \\
Uf & $-6.0 \%$ & $-25.6 \%\left(^{*}\right)$ & $-17.6 \%\left(^{*}\right)$ \\
Uv & $-7.8 \%$ & $-28.8 \%\left(^{*}\right)$ & $-20.8 \%$ \\
Ur & $-2.1 \%$ & $-19.7 \%\left(^{*}\right)$ & $-9.6 \%$ \\
\hline
\end{tabular}

(") Dunnett test: $\mathrm{p}<0.05$.

vs. $\mathrm{T}_{0}$ ); analysing the separate groups, a trend of reduction of skin pigmentation was demonstrated in particular for Group $1\left(-2.2 \% \mathrm{~T}_{20 \mathrm{~min}},-4.9 \% \mathrm{~T}_{2 \mathrm{wks}}\right.$ and $-3.9 \% \mathrm{~T}_{4 \mathrm{wks}}$ vs. $\left.\mathrm{T}_{0}\right)$.

The evaluation of the submental ptosis was performed on frontal images taken in standardized conditions at $\mathrm{T}_{0}$, $\mathrm{T}_{20 \mathrm{~min}}$ and $\mathrm{T}_{4 \mathrm{wks}}$ and submitted to image analysis; in particular the measure considered facial coordinates of each subject in order to identify the relaxed cutaneous area. Results obtained on pooled data and on single study group, did not highlight any significant variation of the facial coordinates.

Regarding the clinical evaluation of the entire population, the study product determined:

- a clinically important and statistically significant improvement of crow's feet and cutaneous microrelief clinical score already 20 minutes after the $1^{\text {st }}$ product application (Dunnett's test $\mathrm{p}<0.05 \mathrm{~T}_{20 \mathrm{~min}}, \mathrm{~T}_{2 \mathrm{wks}}$ and $\mathrm{T}_{4 \mathrm{wks}}$ vs. $\mathrm{T}_{0}$ ), with a percentage of improved subjects at the end of the study (at least one grade of improvement) respectively of $79 \%$ and $76 \%$;

- a statistically significant decrease of nasolabial folds clinical score after 4 weeks of treatment (Dunnett's test $p$ $<0.05 \mathrm{~T}_{4 \mathrm{wks}}$ vs. $\mathrm{T}_{0}$ ) with $52 \%$ of improved subjects;

- a significant improvement of skin dullness clinical score (the skin appears brighter) already 20 minutes after the $1^{\text {st }}$ product application (Dunnett's test $\mathrm{p}<0.05 \mathrm{~T}_{20 \mathrm{~min}}$, 
$\mathrm{T}_{2 \mathrm{wks}}$ and $\mathrm{T}_{4 \mathrm{wks}} \mathrm{vs}$. $\mathrm{T}_{0}$ ), with a percentage of improved subjects (at least one grade of improvement) of $67 \%$ after 4 weeks of treatment;

- a statistically significant improvement of skin tonicity already after 2 weeks of treatment (resistance to traction: Dunnett's test $\mathrm{p}<0.05 \mathrm{~T}_{2 \mathrm{wks}}$ vs. $\mathrm{T}_{0}$ ) and more marked at the end of the study (resistance to pinching, recovery after pinching, resistance to traction: Dunnett test $\mathrm{p}<0.05 \mathrm{~T}_{4 \mathrm{wks}} \mathrm{vs}$. $\mathrm{T}_{0}$ ) with an improvement proportion of at least 1 grade in $48 \%, 61 \%$ and $67 \%$ of subjects, respectively;

- a statistically important reduction (Dunnett's test $\mathrm{p}<$ $0.05 \mathrm{~T}_{20 \mathrm{~min}}, \mathrm{~T}_{2 \mathrm{wks}}$ and $\mathrm{T}_{4 \mathrm{wks}}$ vs. $\mathrm{T}_{0}$ ) of skin dryness starting from the first product application corresponding to a reduction of the clinical score of at least 1 grade, at the end of the study, in $61 \%$ of treated subjects.

Clinical evaluations, carried out at baseline $\left(\mathrm{T}_{0}\right), 20$ minutes after the $1^{\text {st }}$ product application $\left(\mathrm{T}_{20 \mathrm{~min}}\right)$, after 2 $\left(\mathrm{T}_{2 \mathrm{wks}}\right)$ and $4\left(\mathrm{~T}_{4 \mathrm{wks}}\right)$ weeks of treatment, are summarized in the Spiderming ${ }^{\text {TM }}$ graph (Figure 2).

Obtained results underline the anti-aging activity of the study product: in fact, assuming that a larger graph area corresponds to a more advanced state of skin ageing, it is evident that, already after the $1^{\text {st }}$ product application and more consistently after $2\left(\mathrm{~T}_{2 \mathrm{wks}}\right)$ and $4\left(\mathrm{~T}_{4 \mathrm{wks}}\right)$ weeks of treatment, a marked and clinically relevant reduction

Dunnett test: $* \mathrm{p}<0.05 \mathrm{~T}_{20 \min }$ vs. $\mathrm{T}_{0}, \mathrm{~T}_{2 \mathrm{wks}}$ vs. $\mathrm{T}_{0}, \mathrm{~T}_{4 \mathrm{wks}}$ vs. $\mathrm{T}_{0}$

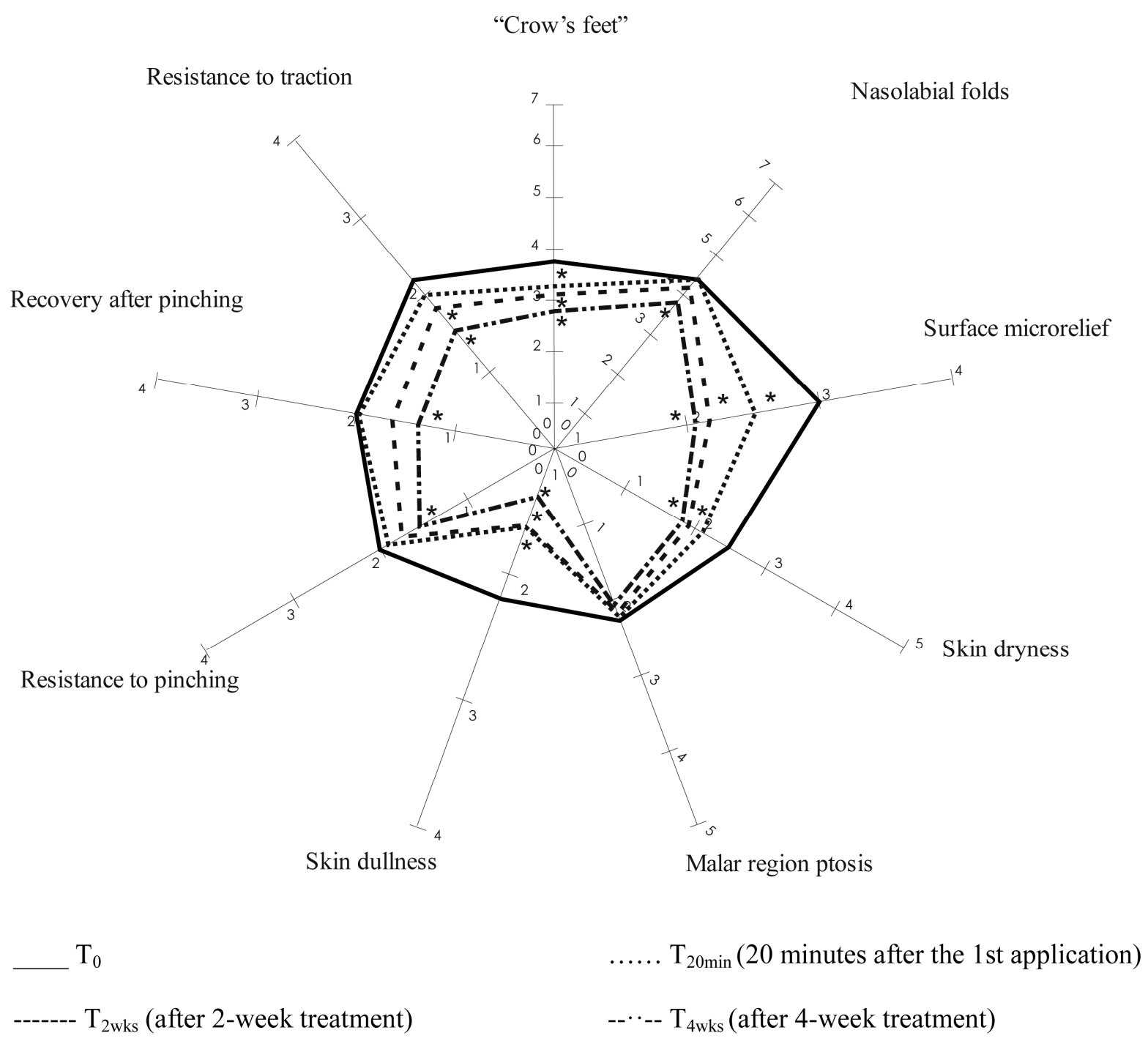

Figure 2. Clinical assessment at different time points on unified data. 
of the most considered clinical signs was achieved.

Looking at the Spiderming ${ }^{\text {TM }}$ graphs for the separated groups (Figures 3-5), the differences among the three study populations at baseline (lower clinical scores for Group 1 and higher for Group 3) and the different responses at the treatment were evident.

In particular for Group 1, a statistically significant decrease of crow's feet clinical score (Dunnett's test $\mathrm{p}<$ $0.05 \mathrm{~T}_{0}$ vs. $\mathrm{T}_{4 \mathrm{wks}}$ ) was present after 4 weeks of treatment with $73 \%$ of improved subjects; moreover, at the end of the study, a clinically important improvement of surface microrelief, resistance to traction (64\% of improved subjects) and of skin dullness (55\% of improved volunteers) were highlighted.

For Group 2 at the end of the treatment, a more marked and statistically significant (Dunnett test $\mathrm{p}<0.05 \mathrm{~T}_{0}$ vs. $\mathrm{T}_{4 \mathrm{wks}}$ ) improvement of the main skin aging signs, was found; crow's feet, surface microrelief, skin dryness, recovery after pinching and skin dullness improved respectively in $73 \%, 82 \%, 82 \%, 82 \%$ and $64 \%$ of volunteers. Moreover, a clinically important improvement of resistance to pinching (on 64\% of subjects) and resistance to traction (on $55 \%$ of subjects) was found. On the oldest population (Group 3), the significant improvement of skin aging clinical signs was even more evident: crow's feet and surface microrelief statistically significantly im-

Dunnett test: $* \mathrm{p}<0.05 \mathrm{~T}_{20 \min }$ vs. $\mathrm{T}_{0}, \mathrm{~T}_{2 \text { wks }}$ vs. $\mathrm{T}_{0}, \mathrm{~T}_{4 \mathrm{wks}}$ vs. $\mathrm{T}_{0}$

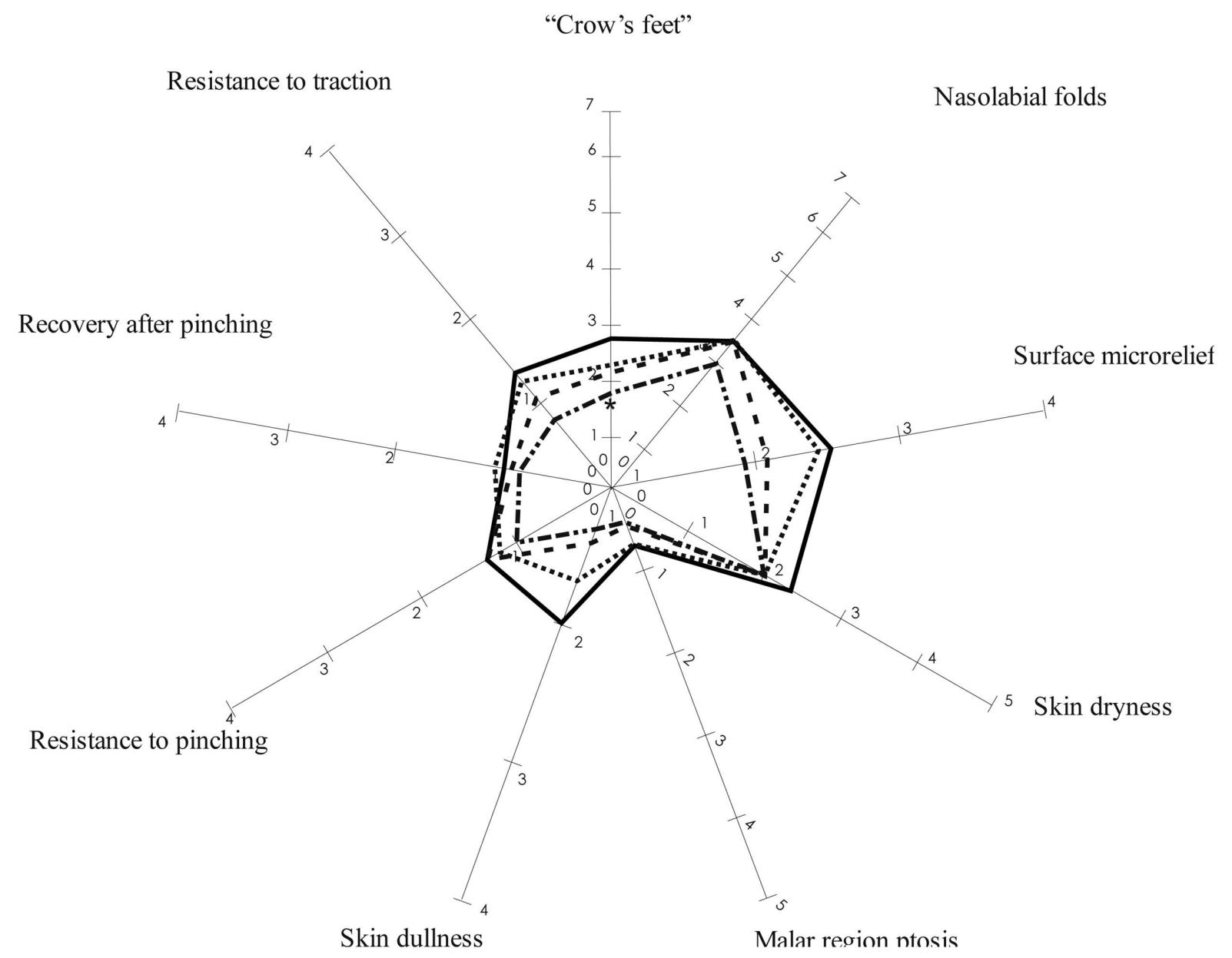

$\mathrm{T}_{0}$

$\ldots . . \mathrm{T}_{20 \min }(20$ minutes after the 1 st application $)$

$\mathrm{T}_{2 \mathrm{wks}}$ (after 2-week treatment)

-- --- $\mathrm{T}_{4 \mathrm{wks}}($ after 4-week treatment)

Figure 3. Clinical assessment at different time points on group 1. 
Dunnett test: $* \mathrm{p}<0.05 \mathrm{~T}_{20 \min }$ vs. $\mathrm{T}_{0}, \mathrm{~T}_{2 \mathrm{wks}}$ vs. $\mathrm{T}_{0}, \mathrm{~T}_{4 \mathrm{wks}}$ vs. $\mathrm{T}_{0}$

\section{"Crow's feet"}

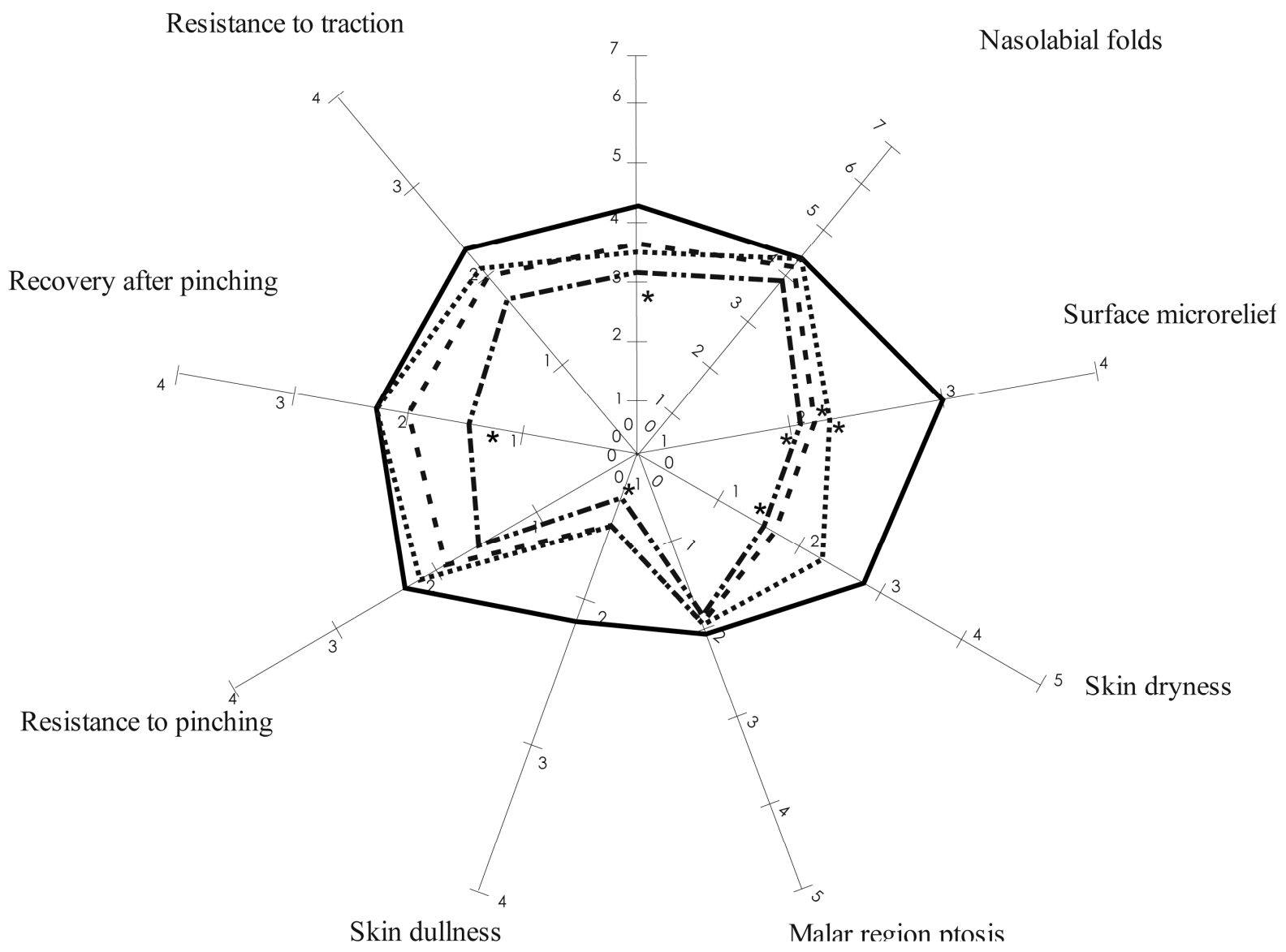

$\mathrm{T}_{0}$

$\ldots . . \mathrm{T}_{20 \min }(20$ minutes after the $1 \mathrm{st}$ application $)$

$\mathrm{T}_{2 \mathrm{wks}}($ after 2-week treatment)

--'-- $\mathrm{T}_{4 \mathrm{wks}}($ after 4-week treatment)

Figure 4. Clinical assessment at different time points on group 2.

proved starting from $\mathrm{T}_{2 \mathrm{wks}}$ (Dunnett's test $\mathrm{p}<0.05 \mathrm{~T}_{2 \mathrm{wks}}$ and $\mathrm{T}_{4 \mathrm{wks}}$ vs. $\mathrm{T}_{0}$ ), with a percentage of improved population respectively of $91 \%$ and $82 \%$, both at $\mathrm{T}_{2 \text { wks }}$ and $\mathrm{T}_{4 \mathrm{wks}}$. Nasolabial folds, skin dullness, recovery after pinching and resistance to traction, significantly improved at the end of the study (Dunnett's test $\mathrm{p}<0.05 \mathrm{~T}_{4 \mathrm{wks}}$ vs. $\mathrm{T}_{0}$ ) on $73 \%$ (nasolabial folds) and $82 \%$ (all the other items) of included subjects. Also the resistance to pinching (55\% of improved volunteers), the malar ptosis ( $45 \%$ of improved subjects) and the skin dryness ( $64 \%$ of improved subjects) showed a clinically important variation at the end of the trial, compared to baseline.
Product cosmetic acceptability resulted good/excellent in the opinion of the subjects enrolled. In particular, a high percentage of volunteers appreciated product colour (good-excellent $=97 \%)$, absorption $($ good-excellent $=$ $97 \%)$, consistency $($ good-excellent $=91 \%$ ), spreadability (good-excellent $=100 \%)$ and the skin feeling (good-excellent $=94 \%$ ). Moreover, the subjects underlined the absence of oiliness (good-excellent $=91 \%$ ) and of product residues (good-excellent $=97 \%$ ). The majority of volunteers $(54 \%)$ did not appreciate the gel perfume before application; however, once applied the product perfume resulted more pleasing $(61 \%=$ good-excellent $)$. 
Dunnett test: ${ }^{*} \mathrm{p}<0.05 \mathrm{~T}_{20 \min }$ vs. $\mathrm{T}_{0}, \mathrm{~T}_{2 \mathrm{wks}}$ vs. $\mathrm{T}_{0}, \mathrm{~T}_{4 \mathrm{wks}}$ vs. $\mathrm{T}_{0}$

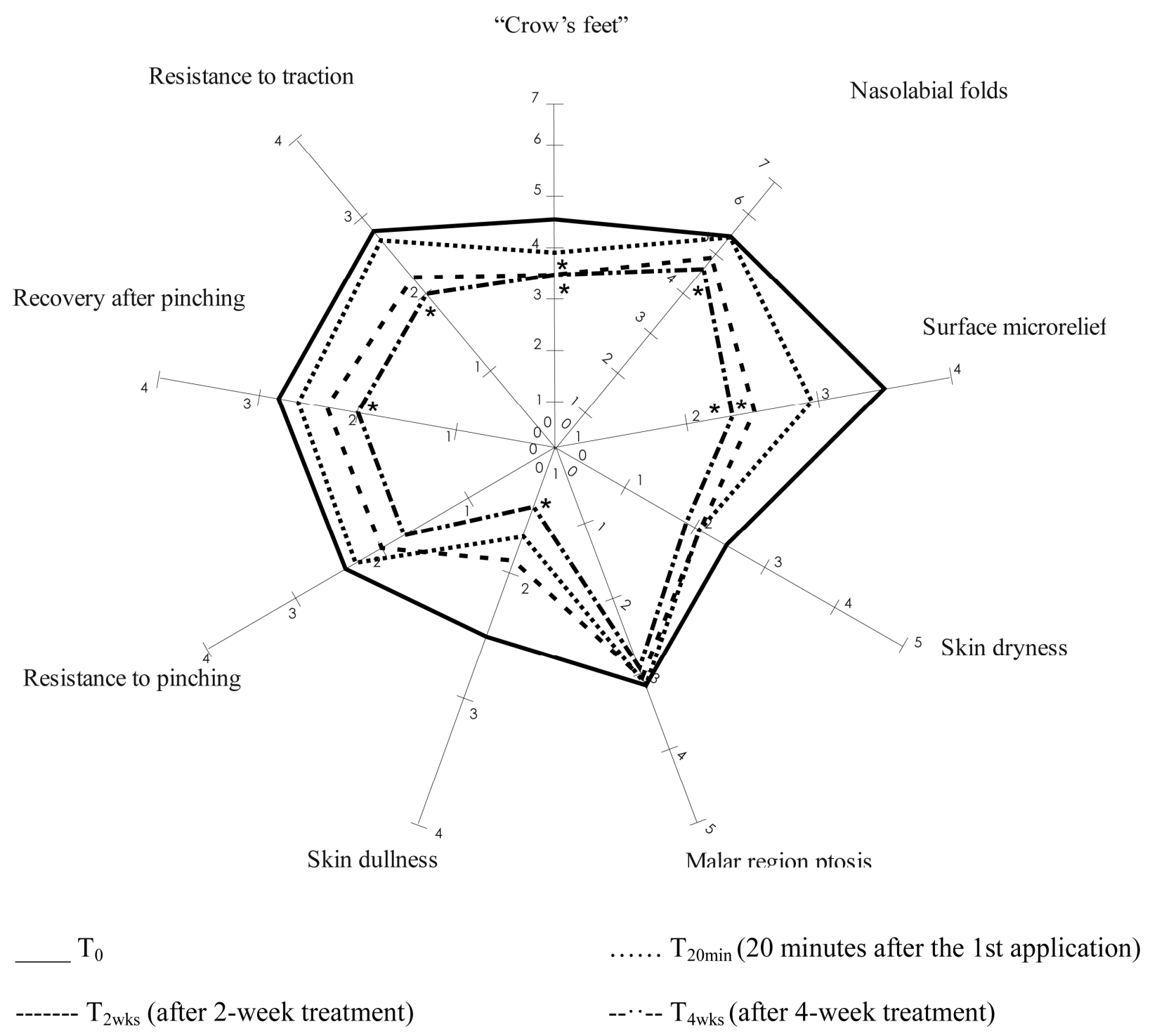

Figure 5. Clinical assessment at different time points on group 3.

At the end of the trial, almost all volunteers appreciated the treatment efficacy. An improvement in skin smoothness ( $27 \%$ medium, $43 \%$ marked and $30 \%$ very marked), $97 \%$ of deep wrinkles ( $45 \%$ medium, $42 \%$ marked and $10 \%$ very marked) and $94 \%$ of superficial wrinkles (36\% medium, $36 \%$ marked and $22 \%$ very marked) was observed in $100 \%$ of subjects, while $58 \%$ of volunteers reported an improvement in face silhouette (37\% medium, $18 \%$ marked and $3 \%$ very marked). Moreover, $91 \%$ of subjects observed an improvement in skin tonicity $(30 \%$ medium, $43 \%$ marked and $18 \%$ very marked), $88 \%$ of skin brightness ( $36 \%$ medium, $42 \%$ marked and $10 \%$ very marked) and $76 \%$ of skin dryness (28\% medium, $21 \%$ marked and $27 \%$ very marked).

All volunteers judged products safety as "good" or "excellent" and these results were further confirmed by the findings of the investigator who, during the entire trial, did not record any type of adverse effect or eventeither related or unrelated to the product use.

\section{Discussion}

Common products used to prevent skin ageing may contain hyaluronic acid which is a natural constituent of the skin and has a certain documented anti-wrinkles activity [13]. Hops extracts are also known for their antioxidant properties and efficacy in improving skin structure and 
firmness has been reported [14]. On the contrary, ethanol was never used before because it tends to cause skin dryness and to remove the superficial lipid film. In the tested formulation, the presence of ethanol was able to synergise with both hyaluronic acid and hops by increasing the effect of these ingredients in a previous in vitro study [15].

This is the first study in vivo on human beings and the obtained results strongly confirm the preliminary in vitro screening, showing an excellent efficacy of the new gel product as anti-skin aging treatment. In fact, the new antiage product gave excellent instrumental and clinical results, showing a statistically and clinically important reduction of skin roughness (smoothing/anti-wrinkle activity), a statistically important and clinically relevant improvement of skin firmness (firming activity), of skin dryness (moisturizing activity), and in general, a statistically significant improvement of global skin rejuvenation (anti-age activity).

The stratification by age of the enrolled subjects led to further considerations: younger skins (Group 1) seem to respond better to the treatment in terms of decrease of roughness of the skin and of maximum decrease of the depth wrinkle. Further on, younger skin had a more evident effect in increasing skin brightness and in reducing skin pigmentation. Conversely, older skin (Group 3) was the most sensitive to the treatment with the new gel in terms of skin hydration, and this was more than expected, as well as of skin firmness, probably induced by reddensifying activity of study product. Subjects belonging to the intermediate age range (Group 2) apparently were those who mostly benefitted from the study treatment: very marked anti-wrinkle, firming and moisturizing activities.

\section{Conclusions}

In conclusion, the skin appeared less wrinkled, smoother, more hydrated, more toned and brighter, therefore generally "younger" looking.

In line with a self-evaluation, the treatment activity was considered as positive by a very high percentage of subjects.

Finally, no adverse events occurred during the trial, showing very good product safety and tolerability, confirmed also by the volunteers.

\section{Conflict of Interest}

The clinical study was 100\% supported by Polichem SA that is the holder of all rights related to the study. F.S., M.C. and F.M. are employees of Polichem SA.

\section{REFERENCES}

[1] I. Ghersetich, M. Troiano, V. De Giorgi and T. Lotti, "Receptors in Skin Ageing and Anti-Aging Agents," Der- matologic Clinics, Vol. 25, No. 4, 2007, pp. 655-662. http://dx.doi.org/10.1016/j.det.2007.06.018

[2] G. J. Fisher, J. Varani and J. J. Voorhees, "Looking older: Fibroblast Collapse and Therapeutic Implications," JAMA Dermatology, Vol. 144, No. 5, 2008, pp. 666-672. http://dx.doi.org/10.1001/archderm.144.5.666

[3] M. Wlaschek, et al., "Solar UV Irradiation and Dermal Photoaging," Journal of Photochemistry and Photobiology B: Biology, Vol. 63, No. 1-3, 2001, pp. 41-51. http://dx.doi.org/10.1016/S1011-1344(01)00201-9

[4] W. Ma, et al., "Chronological Ageing and Photoageing of the Fibroblasts and the Dermal Connective Tissue," Clinical and Experimental Dermatology, Vol. 26, No. 7, 2001, pp. 592-599.

http://dx.doi.org/10.1046/j.1365-2230.2001.00905.x

[5] G. J. Fisher, "The Pathophysiology of Photoaging of the Skin," Cutis, Vol. 75, No. 2S, 2005, pp. 5-9.

[6] L. D. Muiznieks, A. S. Weiss and F. W. Keeley, "Structural Disorder and Dynamics of ELastin," Biochemistry and Cell Biology, Vol. 88, No. 2, 2010, pp. 239-250. http://dx.doi.org/10.1139/O09-161

[7] F. W. Danby, "Nutrition and Aging Skin: Sugar and Glycation," Clinics in Dermatology, Vol. 4, No. 28, 2010, pp. 409-411.

http://dx.doi.org/10.1016/j.clindermatol.2010.03.018

[8] M. F. Demierre, et al., "Public Knowledge, Awareness, and Perceptions of the Association between Skin Aging and Smoking," Journal of the American Academy of Dermatology, Vol. 41, No. 1, 1999, pp. 27-30. http://dx.doi.org/10.1016/S0190-9622(99)70401-9

[9] E. Makrantonaki, et al., "Skin and Brain Age Together: The Role of Hormones in the Ageing Process," Experimental Gerontology, Vol. 45, No. 10, 2010, pp. 801-813. http://dx.doi.org/10.1016/j.exger.2010.08.005

[10] J. K. Rivers, "The Role of Cosmeceuticals in Antiaging Therapy," Skin Therapy Letters, Vol. 13, No. 8, 2008, pp. 5-9.

[11] US Food Drug Administration, "Alpha Hydroxy Acids in Cosmetics," US Food Drug Administration, Silver Spring, 2013.

http://www.fda.gov/Cosmetics/ProductandIngredientSafet $\mathrm{y} /$ SelectedCosmeticIngredients/ucm 107940.htm

[12] A. Sparavigna, M. Setaro and A. Di Pietro, "Healthy Skin 2005: Results of Italian Study on Healthy Population with Particular Regard to the Ageing Phenomenon," Journal of Plastic Dermatology, Vol. 2, 2006, pp. 23-29.

[13] T. Pavicic, et al., "Efficacy of Cream-Based Novel Formulations of Hyaluronic Acid of Different Molecular Weights in Anti-Wrinkle Treatment," Journal of Drugs in Dermatology, Vol. 10, No. 9, 2011, pp. 990-1000.

[14] N. Philips, et al., "Direct Inhibition of Elastase and Matrixmetalloproteinases and Stimulation of Biosynthesis of Fibrillar Collagens, Elastin, and Fibrillins by Xanthohumol," Journal of Cosmetic Science, Vol. 61, No. 2, 2010, 2006, pp. 125-132.

[15] E. Bocchietto, "Polichem Internal Data," 2013. Unpublished. 